\title{
Adherencia al tratamiento antirretroviral como conducta promotora de salud en adultos mayores
}

\section{Adherence to the antiretroviral treatment as a health-promoting behavior in elderly adults}

\section{Adesão ao tratamento antiretroviral como comportamento promotor de saúde em idosos}

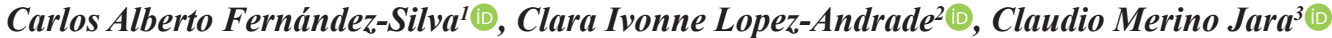



Introducción: La adherencia al tratamiento antirretroviral es una conducta que favorece la salud de las personas que viven con el virus de inmunodeficiencia adquirida; para su logro se debe tener presente las características y experiencias individuales, aspectos cognitivos y de tipo afectivo, conceptos que están establecidos en el Modelo de promoción de la Salud. Objetivo: Analizar la adherencia al tratamiento antirretroviral en un grupo de adultos mayores en un hospital de Chile, en consideración del Modelo de Promoción de la Salud. Materiales y Métodos: Estudio transversal y correlacional que incluyó a 18 adultos mayores, en quienes se analizó algunos conceptos del Modelo de Promoción de la Salud. Se utilizó un instrumento que incluyó el cuestionario simplificado de adherencia a tratamiento (sensibilidad $72 \%$, especificidad del 87\%). Resultados: La adherencia al tratamiento fue de 94,4\%. Los usuarios presentan cogniciones y afectos favorables para la adherencia, siendo la percepción de autoeficacia de un $89,9 \%$. Se encontró asociación entre la adherencia y el dejar de tomar medicamentos ante efectos adversos y/o sentimientos negativos hacia los mismos $(\mathrm{p}=0,001$, Pearson de $-0,714)$, además de ésta con la percepción general de barreras $(\mathrm{p}=0,021 \mathrm{~V}$ de Cramer de -0,542). Discusión: Aspectos como la autoeficacia percibida, la baja percepción de barreras y las motivaciones personales, favorecieron la adherencia al tratamiento. Conclusiones: Los adultos mayores han logrado la adherencia al tratamiento gracias a la percepción de beneficios y de escasez barreras para el acceso al mismo.

Palabras clave: Atención de Enfermería; Cumplimiento de la Medicación; Teoría de Enfermería; Terapia Antirretroviral Altamente Activa; Anciano.

Abstract

Introduction: Adherence to antiretroviral treatment is a behavior that favors the health of individuals living with the acquired immunodeficiency virus; to achieve such, we must bear in mind individual characteristics and experiences, cognitive and affective aspects, concepts established in the Health Promotion Model. Objective: to analyze adherence to the antiretroviral treatment in a group of elderly adults in a Chilean hospital, in consideration of the Health Promotion Model. Materials and Methods: Cross-sectional and correlational study including 18 elderly adults in whom some concepts of the Health Promotion Model were analyzed. The work used an instrument that included the simplified questionnaire of adherence to treatment (sensitivity $72 \%$, specificity of $87 \%$ ). Results: Adherence to treatment was of $94.4 \%$. The users present cognitions and affects favorable for adherence, with the perception of self-efficacy of $89.9 \%$. Association was found between adherence and interrupting the intake of medications upon adverse effects and/or negative feelings toward these $(\mathrm{p}=0.001$, Pearson of -0.714$)$, in addition to this association with the general perception of barriers $(\mathrm{p}=$ 0.021 , Cramer's V of -0.542). Discussion: Aspects, like perceived self-efficacy, low perception of barriers, and personal motivations favored adherence to treatment. Conclusions: Elderly adults have achieved adherence to treatment due to the perception of benefits and scarcity of barriers to access such.

Key words: Nursing Care; Medication Adherence; Nursing Theory; Antiretroviral Therapy, Highly Active; Aged.

Resumo

Introdução: A adesão ao tratamento antiretroviral é um comportamento que favorece a saúde das pessoas que vivem com o vírus da imunodeficiência adquirida; para sua realização, é necessário ter em mente as características e experiências individuais, aspectos cognitivos e afetivos, conceitos que são estabelecidos no Modelo de Promoção da Saúde. Objetivo: Analisar a adesão ao tratamento antiretroviral em um grupo de idosos de um hospital do Chile, tendo em conta o Modelo de Promoção da Saúde. Materiais e Métodos: Estudo transversal e correlacional que incluiu a 18 idosos em quem foram analisados alguns conceitos do Modelo de Promoção da Saúde. Foi utilizado um instrumento que incluiu o questionário simplificado de adesão ao tratamento (sensibilidade de $72 \%$, especificidade de $87 \%$ ). Resultados: A adesão ao tratamento foi de 94,4\%. Os usuários apresentaram cognições e afetos favoráveis à adesão, sendo a percepção de autoeficácia de $89,9 \%$. Encontrou-se associação entre adesão e suspensão da medicação frente aos efeitos adversos e/ou sentimentos negativos em relação aos mesmos $(\mathrm{P}=0,001$, Pearson de $-0,714)$, além disso, com a percepção geral de barreiras $(\mathrm{p}=0,021 \mathrm{~V}$ de Cramer de -0,542). Discussão: Aspectos como autoeficácia percebida, baixa percepção de barreiras e motivações pessoais favoreceram a adesão ao tratamento. Conclusões: Os idosos alcançaram a adesão ao tratamento graças à percepção dos benefícios e à falta de barreiras ao acesso.

Palavras chave: Cuidados de Enfermagem; Adesão à Medicação; Teoria de Enfermagem; Terapia Antirretroviral de Alta Atividade; Idoso.

Como citar este artículo: Fernández-Silva CA, Lopez-Andrade CI, Merino C. Adherencia al tratamiento antirretroviral como conducta promotora de salud en adultos mayores. Rev Cuid. 2018; 9(2): 2201-14. http://dx.doi.org/10.15649/cuidarte.v9i2.522

(c) (i) (8) 2018 Universidad de Santander. Este es un artículo de acceso abierto, distribuido bajo los términos de la licencia Creative Commons Attribution (CC BY-NC 4.0), que permite el uso ilimitado, distribución y reproducción en cualquier medio, siempre que el autor original y la fuente sean debidamente citados. 


\section{INTRODUCCIÓN}

En Chile, la infección por el Virus de la Inmunodeficiencia Humana (VIH), afecta principalmente a los adultos entre 10 s 20 a 49 años, apreciándose el aumento en el número de casos en el rango de edad de los 10 a 19 años, y la tendencia hacia la feminización de la epidemia ${ }^{1}$. Según las cifras reportadas en el 2016 por el Instituto de Salud Pública, en el ámbito nacional se estima que aproximadamente el $4 \%$ de notificaciones por VIH/SIDA corresponde a adultos mayores desde los 60 años, grupo etario al que debe garantizarse servicios de salud que atiendan a sus necesidades, procurando mejorar su acceso a servicios relacionados con dicha condición de salud ${ }^{2}$.

De acuerdo con las cifras referenciadas por el Ministerio de Salud de Chile, en los adultos mayores se ha observado un aumento considerable en la notificación de casos de VIH/ SIDA, pasando de una tasa de 6,3 por cien mil habitantes en el rango etario de los 60 a 69 años en el período comprendido entre 1988 y 1992, a 23,5 casos por cien mil habitantes en el período 2008-2012, situación que también se puede apreciar en la población de 70 y más años, en los cuales las tasas pasaron de 1,8 casos por cien mil habitantes a 5,4 por cien mil habitantes en el mismo período de tiempo ${ }^{3}$.

Como causa de esta situación, se identifican aspectos como: la prolongación de la vida resultante del tratamiento antirretroviral, la adopción de conductas de riesgo tal como el sexo sin protección, y la vulnerabilidad que se genera por la forma en que los adultos mayores reciben información para su cuidado y cómo la aplican a su vida cotidiana, la percepción social frente a la sexualidad en esta etapa de la vida, además del poco interés por la implementación de medidas sanitarias para combatir la epidemia del VIH en dicha población ${ }^{1,4}$.

Con el fin de lograr la atención integral de sus necesidades sanitarias, es necesario tener presente los componentes biopsicosociales que les son propios, es así como sumado al interés de la compensación biológica evidenciado carga viral indetectable y linfocitos CD4 en número adecuado para brindar inmunidad, se debe tener en consideración otros aspectos que afectan su salud como lo son los estigmas frente a la seropositividad, precepciones sobre su condición, la dificultad de encontrar pareja, formas de prevenir la transmisión, y condiciones de fragilidad asociados o resultantes de la infección, entre otros aspectos, los cuales pueden incidir en su deseo de mantener o mejorar su adherencia al tratamiento ${ }^{6,7}$.

Sumado a lo anterior, se debe tener presente que aspectos como la etapa de la infección, la adaptación de los horarios de los medicamentos a los estilos de vida del individuo, la percepción respecto de los efectos adversos, las dificultades para la identificación de los medicamentos, el tiempo en tratamiento, las barreras institucionales para la atención y la comunicación con el equipo de salud también pueden afectar el logro de la adherencia $^{8-11}$.

Debe tenerse en consideración que los usuarios adultos mayores con VIH, pueden verse enfrentados a la omisión de las dosis de los medicamentos como consecuencia de las alteraciones de la memoria reciente, aspecto 
que pese a ser parte de los cambios esperados en esta etapa, también puede afectarse de forma importante como consecuencia de la infección ${ }^{12}$, trayendo como consecuencia el riesgo de resistencia viral a los medicamentos; esta situación amerita que el personal sanitario cuente con la formación para detectar y prevenir las alteraciones cognitivas que acompañan a los adultos mayores con VIH, con lo cual se aporta al abordaje integral.

Identificados los factores que inciden en el resultado conductual de la adherencia, es pertinente mencionar que para la presente investigación, se tuvo en consideración la definición de la Organización Panamericana de la Salud, desde la cual se establece que ésta corresponde a la capacidad del paciente para implicarse correctamente en la elección, inicio y cumplimiento del mismo a fin de conseguir una adecuada supresión de la replicación viral ${ }^{8}$; al vincular este concepto con los adultos mayores, permite posicionarlos como agentes activos en el manejo de su condición, fomentado así su autovalencia y participación. Desde este contexto, se considera pertinente la aplicación de aspectos disciplinares como las que otorgan los modelos teóricos en enfermería, con el fin de facilitar la sistematicidad en el abordaje de los usuarios, y la implementación de medidas de intervención, seguimiento y evaluación que favorezcan su bienestar.

Según lo anteriormente expuesto y en concordancia con la temática de la investigación, se consideró pertinente adoptar la matriz teórica del Modelo de Promoción de la Salud (MPS), propuesto por la enfermera estadounidense Nola J. Pender en 1982, y que fue actualizado el año 2006 ${ }^{13}$; este modelo toma como bases teóricas los postulados de Albert Bandura, en los cuales se destaca la importancia de los procesos cognitivos para el cambio de conducta; la teoría del aprendizaje social o teoría cognitiva social que incluye los conceptos de autoatribución, autoevaluación y autoeficacia, siendo esta última un parámetro central en el modelo de Pender. Otros referentes son el modelo de valoración de expectativas de la motivación de humana (Feather, 1982) y el modelo de creencia en la salud (Becker, 1974), diferenciándose de éste último en que el miedo o la amenaza no se encuentran con factores de motivación para el logro de una conducta promotora de salud ${ }^{13}$.

A continuación, se presentan sus principales conceptos y definiciones: ${ }^{13,14}$.

Conducta previa relacionada: Se valora en el individuo su actuación frente a la situación abordada o alguna similar que haya enfrentado con anterioridad.

> Factores personales: Los cuales pueden ser biológicos (Ejemplo: la edad o el sexo), psicológicos (como la autoestima y concepto de salud), y socioculturales (etnia, nivel educativo, posición socioeconómica).

$>$ Beneficios percibidos de la acción: los cuales generan motivación para adquirir una conducta promotora de salud.

$>$ Barreras para la acción: las cuales pueden ser personales, interpersonales o ambientales.

La autoeficacia: considerado como un sistema que permite a los individuos percibir, regular, y evaluar la conducta permitiendo la autorregulación de las acciones, sentimientos y pensamientos. 
> Afecto relacionado con la actividad: Corresponde a los sentimientos que se generan antes, durante o después de la conducta promotora de salud, y guardan una relación directamente proporcional con la autoeficacia.

$>$ Influencias interpersonales: Se toma en consideración los referentes y apoyos que posee el individuo, personas más importantes o consideradas como modelos, y sus fuentes de apoyo social y emocional.

> Influencias situacionales: tiene relación con la percepción del individuo frente a situaciones o contextos que pueden influir en su conducta.

$>$ Compromiso con un plan de acción.

$>$ Demandas y preferencias contrapuestas. Las demandas contrapuestas son acciones contrarias a la conducta promotora de salud y que el individuo asume, ya que tiene poco control de las mismas; mientras que, en las preferencias contrapuestas, el individuo escoge voluntariamente una conducta contraria a la conducta promotora de salud.

Para la aplicación de este modelo es importante tener en cuenta que debe haber claridad frente a la conducta promotora de salud a la que se quiere llegar, por ejemplo, si esta corresponde a la adherencia al tratamiento antirretroviral como resultado conductual, en la valoración del individuo deberá incluirse aspectos como ${ }^{13,14}$ :

- La conducta previa de consumo de medicamentos.

- La capacidad que el usuario considera tener para llevar a cabo el tratamiento, aspecto que está ligado a la percepción de autoeficacia.
- Los factores personales que puedan afectar la misma, entre los cuales se encontraría el déficit cognitivo, o aspectos físicos que dificulten la apertura de frascos $y$ organización de los medicamentos.

- Los sentimientos que trae consigo el llevar a cabo la terapia farmacológica ó acudir en forma periódica a la institución de salud.

- La percepción de barreras como los horarios de atención, o facilidad de acceso a los medicamentos.

Estos y otros aspectos relacionados con los conceptos del Modelo teórico de Pender, permitirán establecer un panorama más amplio sobre la posibilidad de logro de la conducta esperada, facilitando así la planificación de intervenciones preventivas y de apoyo contextualizada a las necesidades de los usuarios.

De acuerdo con lo anterior, se estableció como objetivo general para la presente investigación: analizarlaadherenciaal tratamiento antirretroviral como conducta promotora de salud en usuarios adultos mayores del programa de VIH de un hospital público en Chile, y como objetivos específicos: establecer los factores personales (biológicos, psicológicos y socioculturales) de los usuarios; determinar generalidades sobre su condición clínica y respecto de la adherencia al tratamiento como conducta promotora de salud; describir las cogniciones y afectos específicos de la conducta respecto a la terapia antirretroviral, y por último, relacionar las variables en consideración de la adherencia como conducta promotora de salud. 


\section{MATERIALES Y METODOS}

Tipo de estudio: Cuantitativo de corte transversal, con análisis descriptivo y correlacional, el cual se acoge a los lineamientos de la declaración de STROBE (Strengthening the Reporting of Observational Studies in Epidemiology) ${ }^{15}$.

Población de estudio: La institución en la cual se realizó el estudio entregó inicialmente un reporte por base de datos de 30 personas de 60 años y más, sin embargo, tras la actualización de la misma, se obtuvo un listado de 22 adultos mayores vigentes en el programa, de los cuales 18 participaron en el estudio tras aplicar los criterios de inclusión y exclusión.

\section{Criterios de inclusión y exclusión: Se consideró} que para ser incluidos en el estudio, los adultos mayores debían contar con la capacidad de decidir su participación en forma autónoma, motivo por el cual se excluyeron 4 usuarios, ya que presentaban trastornos evidentes de dicha capacidad, debido a secuelas de accidente vascular, demencia ó por encontrarse en condición de hospitalización con alteración del estado de conciencia.

\section{Procedimientos y métodos de recolección} de datos: En forma previa a la recolección de la información, se desarrolló el proceso de consentimiento informado, contándose con la aceptación del total de los participantes, a quienes se procedió a aplicar mediante entrevista, el instrumento elaborado para la investigación.

Dicho instrumento contenía preguntas de elaboración propia por los autores, las cuales estaban relacionadas con conceptos específicos del Modelo de Promoción de la Salud, estos componentes fueron sometidos a prueba de expertos y a pruebas de confiabilidad, arrojando un índice de confiabilidad del 0,81. Se incorporó además el cuestionario simplificado de adherencia a tratamiento ó como se le conoce más cotidianamente por su sigla en inglés: SMAQ (simplified medication adherence questionnaire), el cual reporta una sensibilidad del 72\% y una especificidad del 87\%; y los test de Yesavage para el tamizaje de depresión, y el test Minimental para el tamizaje de deterioro cognitivo. Tanto el instrumento SMAQ como los de valoración cognitiva, se encuentran validados y son aplicados en forma rutinaria por indicación del Ministerio de Salud de Chile ${ }^{1,5}$.

\section{El instrumento resultante contó con tres secciones:}

- Sección I: En la que se evaluó los factores personales del modelo (datos biológicos, psicológicos y socioculturales). Para la valoración de aspectos biológicos, se tuvo presente incluir los datos clínicos de carga viral y CD4, etapa de la enfermedad, esquema de tratamiento antirretroviral y otras patologías diagnosticadas, especificando si debido a ello tenía consumo de otros medicamentos; como parte de la valoración psicológica se tuvo como referente el test de Yesavage y Test Minimental (valoración cognitiva); para los demás ítemes se emplearon preguntas de selección, con posibilidad de ampliación de la respuesta.

- Sección II: Correspondió a la valoración de la adherencia al tratamiento a través del SMAQ.

- Sección III: Permitió valorar las cogniciones y afectos específicos de la conducta relacionados con la adherencia al tratamiento, 
empleando preguntas de selección con posibilidad de ampliación de la respuesta, tal como en la primera sección.

Para la interpretación de adherencia por del test del SMAQ se tuvo en cuenta clasificar a los usuarios como no adherentes cuando respondían positivamente a una o más de las tres primeras preguntas dicotómicas del test (1. ¿Ha olvidado alguna vez tomar sus medicamentos?, 2. ¿Ha descuidado el horario de los medicamentos?, 3. ¿Deja de tomar los medicamentos en los momentos en que se siente mal?) y/o cuando frente a las preguntas 4,5, y 6 (4. En la última semana ¿cuántas veces ha olvidado la terapia?, 5. ¿Olvidó algún medicamento el pasado fin de semana?, 6. En los últimos 3 meses ¿cuántos días no ha tomado toda la terapia?), el usuario respondió: > a 2 dosis la semana pasada o $>$ a 2 días completos sin terapia en los últimos 3 meses. Se tuvo en consideración además, la interpretación del parámetro semicuantitativo, para el cual se toma como referente la pregunta 4 , en la que se evalúa el número de dosis no consumidas en la última semana, siendo: ningún olvido un $95-100 \%$ de cumplimento, 1-2 dosis: 85-94\%, 3-5 dosis: 65 a 84\%, 6-10 dosis: entre el 30 al 64\%, y más de 10 dosis: menos del $30 \%)^{1}$.

Respecto del test de Yesavage, se tuvo en cuenta la interpretación del según los lineamientos del Ministerio de Salud: 0 a 5 puntos: adulto mayor normal, y de 6 o más puntos: depresión de intensidad creciente 5 , no obstante, para la presente investigación los términos de interpretación fueron adaptados a "normal" para la primera clasificación, y "alterado" para la segunda.
Para la interpretación del minimental test (MMSE) se consideró como normal un puntaje $\geq$ a 14 Puntos o alterado, si era $\leq$ a 13 , los cuales son referentes en el contexto nacional ${ }^{5}$.

Variables: Las variables se agruparon según los conceptos del Modelo de Promoción de la Salud, estableciendo la adherencia al tratamiento como la conducta promotora de salud y principal variable de medición. Las demás variables se distribuyeron en las dimensiones de: cogniciones y afectos específicos relacionados con la adherencia, y factores personales, ésta última dimensión incluyó los aspectos de tipo biológico, psicológico y sociocultural.

Análisis de datos: La información recolectada se ingresó en una plantilla de Excel, la cual posteriormente se ingresó en el programa SPSS versión 17 para realizar el análisis descriptivo y correlacional.

Consideraciones éticas: En su fase de proyecto de investigación, se contó con la aprobación del Comité Ético Científico del Servicio de Salud de la región (autorización 47-2016). Como medidas éticas declaradas y aplicadas en la investigación, se desarrolló el proceso de consentimiento informado el cual concluyó en la firma del acta de consentimiento por parte de los participantes con la presencia de un ministro de fe; en dicho documento se dejó claridad respecto de la garantía de confidencialidad frente a la información recolectada y al carácter anónimo de los datos al ser ingresados en una base de datos general. Adicional a lo anterior, se entregó una hoja de información al participante, la cual incluía la información general de la investigación, y los datos de contacto tanto de los investigadores como del Comité Ético Científico. 


\section{RESULTADOS}

Considerando el concepto de factores personales del Modelo de Promoción de la Salud, en lo que respecta a los de tipo biológico, se encontró que el promedio de edad de los adultos mayores fue de 65 años, fluctuando entre los 65 y 75 años; en cuanto al sexo, el 55,6\% son de sexo masculino, mientras que el $44,4 \%$ del sexo femenino.

En cuanto a los factores personales de tipo psicosocial, se encontró que el $83,3 \%$ cuenta con apoyo familiar $\mathrm{y} / \mathrm{o}$ de amistades desde el diagnóstico (DE 1,57, curtosis -0,069, Chi²: 0,011), destacándose que el 16,7\% aún mantiene en reserva su condición. Se pudo apreciar que el 94,4\% presentaban un Minimental test normal (DE 0,23, curtosis $18, \mathrm{Chi}^{2}: 0,000$ ) y el $5,6 \%$ presentó alteraciones, teniendo presente que dichas alteraciones se relacionaron con el nivel educativo del participante. El tamizaje de depresión mediante test de Yesavage evidenció que el $72,2 \%$ estaban dentro de lo normal, mientras que el $27,8 \%$ se clasificó en una depresión leve.

Se encontró que el 55,6\% tienen pareja o están casados, mientras que el $44,4 \%$ se clasifican como soltero sin pareja o en condición de viudez; el $72,2 \%$ tiene hijos, con un promedio de 2,5 hijos por usuario/a; el 83,3\% vive con familiares (DE 1,09, curtosis 6,36, Chi²: 0,000); el 11,1\% vive solo y 5,6\% en una residencia. El 88,9\% realiza alguna práctica religiosa, predominando la católica con un 44,4\% y la evangélica con un $22,2 \%$. Respecto de la situación laboral, los usuarios perciben ingresos bien sea por pensión (77,8\% DE 1,53, Curtosis 0,012, Chi²: 0,011), o porque aún se encuentran laboralmente activos (empleados 11,1\% y trabajador independiente $11,1 \%$ ). Se destaca que el $88,9 \%$ de los usuarios tienen algún nivel educativo, mientras que el $11,1 \%$ no posee ninguna educación formal.

Al indagar sobre el pertenecer a grupos organizados, se encontró que el 61,1\% no acude a alguno de éstos, mientras que el 38,9\% sí lo hace, siendo los grupos de iglesia los más frecuentados e $57,1 \%$, seguido por los grupos de adulto mayor $28,6 \%$ y otro tipo de agrupaciones con un $14,3 \%$. Se identificó entre las causas para no pertenecer a estas organizaciones con un $63,6 \%$, no desear realizarlo porque "no le gusta ese tipo de actividades", mientras que el 36,4\% mencionó que se debía a dificultades por el trabajo, el desconocimiento frente al funcionamiento de los grupos y/o por domicilio lejano.

Respecto de las generalidades del tratamiento y la adherencia al mismo, se estableció que el $100 \%$ de los evaluados se encuentran en etapa A, destacándose que el 88,9\% presentan una carga viral indetectable ( $\mathrm{Chi}^{2}$ : 0,000$)$, y un promedio de CD4 $500 \mathrm{cel} / \mathrm{mil}$; lo cual indica estabilidad clínica. Se evidenció que el 66,6\% llevan hasta 5 años de tratamiento, seguido por el 33,3\% quienes llevan 6 o más años, siendo 12 años el tiempo máximo registrado por un usuario. Además del diagnóstico de VIH, el 77,8\% de los usuarios presentan otras patologías, entre las que se destacan las de tipo cardiovascular como la hipertensión arterial, la diabetes y dislipidemia.

En cuanto al consumo de medicamentos, se pudo apreciar que el 83,3\% de los usuarios se encuentra en condición de polifarmacia, con un 33,3\% que consumen 3 medicamentos al día, seguido por $22,2 \%$ quienes consumen hasta 6 medicamentos. 
El 61,1\% de los usuarios no lleva ningún método para organizar sus medicamentos (DE 1,42, curtosis: 1,032, $\mathrm{Chi}^{2}$ : 0,002), y del 38,9\% que si lo hacen, refieren que emplean en su mayoría pastilleros, aunque también se evidenció el uso de recordatorio por familiares, amigos o cuidadores y el uso de las alarmas de celular; se destaca que existe continuidad en el retiro de los medicamentos en un $94,4 \%$ de los usuarios.
En la evaluación de adherencia al tratamiento con el SMAQ se pudo apreciar que el 55,5\% es adherente, no obstante en la evaluación semicuantitativa del mismo instrumento, se pudo establecer que existe adherencia en el 94,4\%; adicional a esta valoración, se les preguntó a los usuarios si desde su percepción se consideraban adherentes al tratamiento explicándoles previamente en qué consiste dicho concepto, con lo cual se logró establecer desde la percepción subjetiva, que el $100 \%$ se consideró adherente Figura 1.

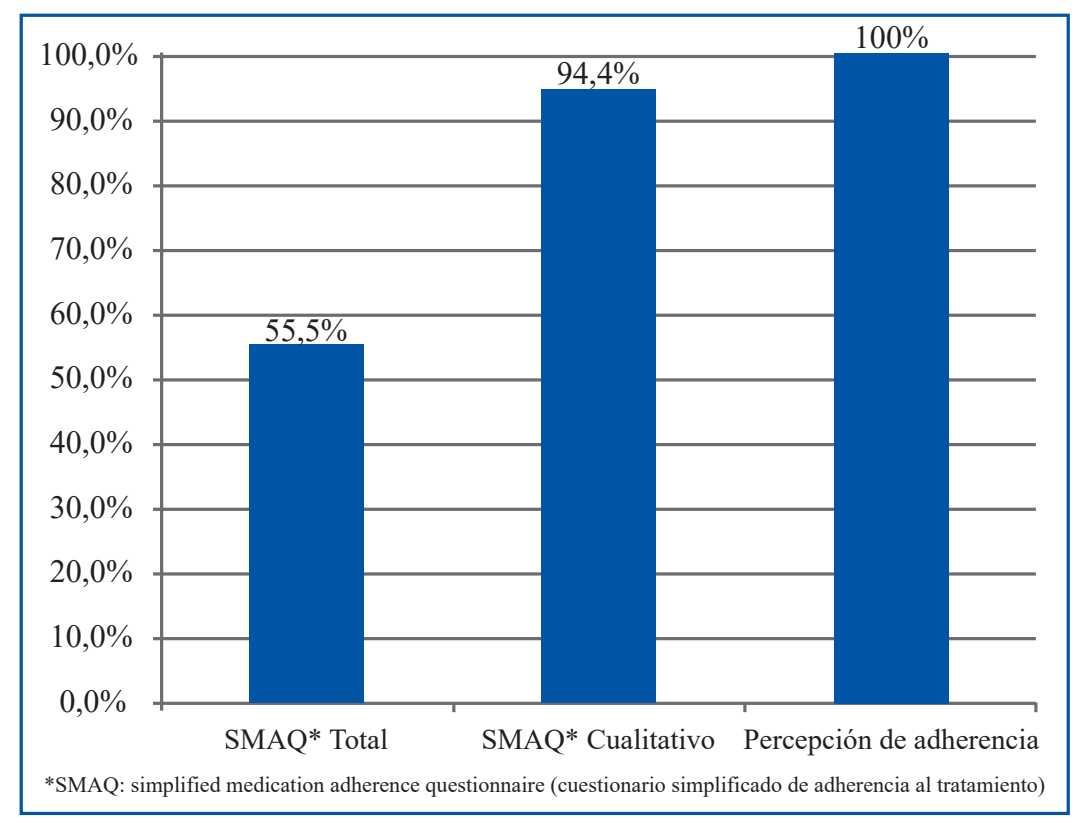

Figura 1. Interpretación de la adherencia por el test de SMAQ (simplified medication adherence questionnaire) y percepción subjetiva del usuario

Fuente: Elaboración propia de los autores.

En consideración a la estabilidad clínica que presentan los usuarios, se tomó como referencia para el análisis correlacional de la adherencia, los resultados de la evaluación semicuantitativa del SMAQ, no obstante, el que algunos hayan manifestado que dejan de tomar sus medicamentos en forma ocasional.
En consideración al Modelo de Promoción de la Salud se abordaron aspectos relacionados con las cogniciones y afectos relacionados con la conducta, considerando esta como el consumir el tratamiento antirretroviral, se encontró que el $88,9 \%$ de los usuarios refieren que el tratamiento es benéfico para su salud, en contraste con el $11,1 \%$ quienes manifestaron que no, siendo el 
motivo para ello la aparición de eventos adversos de los medicamentos y el temor que estos se puedan presentar en el largo plazo.

Pese a que la investigación se planteó bajo el diseño cuantitativo, se consideró importante mencionar los siguientes aspectos cualitativos referidos por los como parte de sus sentimientos frente a la terapia: “en algún momento le pasarán la cuenta al estómago", y una usuaria opinó que los medicamentos "la dejan mal", afirmando además: “(...) antes era más sana, no soy la misma desde que tomo el tratamiento, tengo menos energía (...)".

El 83,3\% refiere que no percibe alguna barrera para llevar a cabo su tratamiento, mientras que el 16,7\% sí, refiriendo como causas: la sensación de agotamiento frente al consumo de medicamentos, los efectos adversos de tipo gastrointestinal, los olvidos de las dosis y la falta de dinero para el desplazamiento.

Al indagar respecto de los sentimientos que le genera el hecho de consumir los medicamentos, se pudo apreciar que el $88,9 \%$ refirió que estos son positivos, mientras que el 11,1\% manifestó que eran negativos, siendo estos principalmente: el enojo, la frustración ó tristeza. El 88,9\% evidencia tener motivaciones para realizar su tratamiento destacándose que el mantenerse bien, evitar la enfermedad y la motivación que le generan sus familiares y amigos son los principales aspectos para ello; se destaca que el 11,1\% de los usuarios evaluados comentó que no tiene alguna motivación para realizar el tratamiento. El 83,3\% consideran que tienen un modelo a seguir entre sus familiares cercanos o amistades, que les permite mantenerse adherentes, aspecto que resulta relevante al momento de planificar intervenciones educativas.

Según los objetivos establecidos y tras el análisis correlacional lineal, se identificaron resultados representativos en los factores personales, tanto socioculturales, psicológicos y biológicos, estos últimos relacionados con la condición clínica.

Al evaluar los factores personales socioculturales, el contar con un grupo organizado presentó asociación con las variables: contar con un modelo a seguir ( $\mathrm{p}=0,008$ Pearson de 0,884), y tiempo de tratamiento $(\mathrm{p}=0,032$ Pearson de 0,798); respecto de los aspectos psicológicos, se encontró asociación entre el resultado de minimental y la continuidad en el retiro de medicamentos $(\mathrm{p}=<$ 0,001 Pearson 1,0) Ver Tabla 1. 
Tabla 1. Correlaciones mediante coeficiente de Pearson en las variables del estudio

\begin{tabular}{|c|c|c|c|}
\hline Variable 1 & Variable 2 & $\begin{array}{c}\text { Coeficiente de } \\
\text { correlación }\end{array}$ & Valor de p \\
\hline Modelo a seguir & Grupo Organizado &, 884 & 0,008 \\
\hline Tiempo en Tratamiento & Grupo Organizado &, 798 & 0,032 \\
\hline Clasificación MMSE & Continuidad en el Retiro & 1,000 & $<0,001$ \\
\hline Beneficios & CD4 &, 739 & $<0,001$ \\
\hline $\begin{array}{c}\text { Deja de tomar medicamentos } \\
\text { si se siente mal }\end{array}$ & Adherencia por SMAQ \% &,- 714 & 0,001 \\
\hline Sentimiento Negativo & Adherencia por SMAQ \% &,- 714 & 0,001 \\
\hline $\begin{array}{c}\text { Olvidó sus medicamentos } \\
\text { la última semana }\end{array}$ & SMAQ \% & 1,000 & $<0,001$ \\
\hline $\begin{array}{c}\text { Olvidó sus medicamentos } \\
\text { la última semana }\end{array}$ & Sentimiento Negativo &,- 714 & 0,001 \\
\hline $\begin{array}{c}\text { Deja de tomar medicamentos } \\
\text { si se siente mal }\end{array}$ & Barreras &, 791 & $<0,001$ \\
\hline
\end{tabular}

Fuente: Elaboración propia de los autores.

En cuanto a los aspectos personales de tipo biológico, se encontró asociación entre la percepción de beneficios respecto del tratamiento y el valor de CD4 $(\mathrm{p}=<0,001$, Pearson de $0,739)$, de igual manera se encontró correlación inversa entre el dejar de tomar medicamentos y la presencia de sentimientos negativos, con la adherencia al tratamiento $(\mathrm{p}=0,001$, Pearson de $-0,714)$ Ver Tabla 1.

Además de lo anterior, se encontró correlación entre el olvido de dosis en la última semana, y dos variables: la adherencia por SMAQ $(<0,001$, Pearson: 1,000) y la presencia de sentimientos negativos, la cual arrojó una correlación inversa $(p=0,001$ Pearson: -0,714) Ver Tabla 1 .

Con respecto a la Adherencia al tratamiento antirretroviral y en relación con el Modelo de Promoción de la Salud (MPS) se encontraron asociaciones con: la percepción de barreras $(p=0,021 \mathrm{~V}$ de Cramer de -0,542), y las motivaciones y consumo pese a cualquier circunstancia $(\mathrm{p}=0,004 \mathrm{~V}$ de Cramer de 0,686) Ver Tabla 2.

Tabla 2. Prueba de independencia definitiva entre la adherencia al tratamiento y variables del Modelo de Promoción de la Salud

\begin{tabular}{|l|c|c|c|c|c|c|c|}
\hline $\begin{array}{l}\text { Adherencia Vs } \\
\text { Variables MPS }\end{array}$ & $\mathbf{C h i}^{2}$ & $\mathbf{p}<\mathbf{0 , 0 5}$ & Estadístico & Valor & $\begin{array}{c}\text { Estimación } \\
\text { de Riesgo }\end{array}$ & Mínimo & Máximo \\
\hline Barreras & 5,29 & 0,021 & V de Cramer & $-0,542$ & 0,66 & 0,3 & 1,48 \\
\hline Motivaciones & 8,47 & 0,004 & V de Cramer & 0,686 & 2 & 0,5 & 7,99 \\
\hline $\begin{array}{l}\text { Consumo pese a } \\
\text { cualquier circunstancia }\end{array}$ & 8,47 & 0,004 & V de Cramer & 0,686 & 2,00 & 0,5 & 7,99 \\
\hline
\end{tabular}

MPS: Modelo de Promoción de la Salud.

Fuente: Elaboración propia de los autores. 


\section{DISCUSIÓN}

Los factores personales de tipo biológico de los adultos mayores participantes en el estudio, corresponden al perfil reportado a nivel nacional, en los cuales se destaca la predominancia del sexo masculino para usuarios con VIH en este grupo etario $2,3,16$.

En cuanto a los factores personales de tipo psicosocial, se pudo apreciar la familia es quien brinda apoyo emocional y compañía, siendo este un parámetro relevante al ser favorecedor de la adherencia al tratamiento, lo cual ha sido reportado en otros estudios ${ }^{17,18}$. Este dato debe ser considerado en la planificación de actividades educativas, ya que, éstas tendrán mayor efectividad si se involucra a las personas que sean significativas, quienes desde el Modelo de Promoción de la Salud, estarían catalogados como influencias de tipo personal para el logro de la conducta promotora de salud ${ }^{13}$.

Es de mencionar que los resultados obtenidos en cuanto a la participación comunitaria, aspectos laborales, prácticas religiosas y demás aspectos de tipo social, coinciden con aquellas descritas para los adultos mayores a nivel país ${ }^{19}$.

Peseaqueno seencontraron referencias específicas para comparar los resultados de evaluación cognitiva y de depresión, se puede mencionar que estos distan de lo esperado para personas con las características de los participantes ${ }^{7,20}$; y muy similares en cuanto al tamizaje de depresión al reportado por la literatura para usuarios con la misma condición pero pertenecientes a diferentes grupos etarios, en los cuales estaban incluidos los adultos mayores ${ }^{21,22}$; esta situación podría explicar la asociación existente entre el estado mental de los participantes y el retiro constante de sus medicamentos.

Respecto de los factores personales de tipo sociocultural, es de mencionar que el hecho de que los adultos mayores refieran participar en actividades comunitarias y que a su vez se relacione con tener un modelo a seguir, es decir, que tengan una percepción positiva frente a la satisfacción social, facilita el logro de la conducta promotora de salud ${ }^{12}$, aspecto que de no estar presente, representa un factor que incide negativamente en la adherencia al tratamiento ${ }^{17}$.

Se puede afirmar entonces, que el perfil general del adulto mayor con VIH, coincide con el perfil reportado en el ámbito nacional para los adultos mayores con diferentes condiciones de salud asociadas $^{22}$, y que el perfil biológico y psicosocial coincide con los descritos para poblaciones de adultos y adultos mayores con diagnóstico de $\mathrm{VIH}^{23-25}$.

Pese a la estabilidad clínica reportada en los participantes, se pudo evidenciar que poseen otras patologías asociadas de tipo crónico, como lo son la hipertensión y diabetes, lo cual difiere de los resultados de otros estudios en los cuales las enfermedades prevalentes en personas con las mismas características, eran las de tipo infeccioso ${ }^{25}$, sin embargo, es de destacar que dicha condición favorece la aparición del síndrome geriátrico de polifarmacia, caracterizado en esta población por el consumo de hasta 6 medicamentos al día, lo cual se asemeja a las evidencias reportadas respecto de dicho síndrome ${ }^{26-28}$. 
Es de destacar que en su mayoría, los usuarios mencionaron no llevar métodos para organizar sus medicamentos, y pese a la polifarmacia, se encuentran clínicamente estables y en su mayoría reportan adherencia al tratamiento antirretroviral, aspectos que se ven limitados para la discusión, debido a que no fue sido posible encontrar evidencia al respecto para poblaciones de adultos mayores que viven con VIH.

Es de tener presente que pese al reporte mayoritario de adherencia, el que algunos participantes hayan manifestado que dejan de tomar sus medicamentos en forma ocasional es un riesgo de resistencia a la medicación a largo plazo, y por ende de falla virológica ${ }^{29}$, lo cual los aleja del logro o mantención de la conducta promotora de salud, por lo tanto es un parámetro que debe ser intervenido en forma constate a través de actividades educativas y de seguimiento ${ }^{30}$, tal como se realiza en otras patologías crónicas no transmisibles ${ }^{31}$.

Dentro de las cogniciones y afectos específicos de la conducta se encontró que los usuarios se sienten en capacidad de llevar a cabo su tratamiento, es decir, que tienen una percepción favorable de autoeficacia, y a su vez baja percepción de barreras, sin embargo, se pudo identificar en quienes manifestaron barreras, una asociación estadística negativa con la adherencia al tratamiento; estos aspectos han sido descritos como trascendentales en el resultado de la adherencia $^{8,13,23}$.

Como parte de los afectos, se pudo identificar en la mayoría de los participantes que el consumir el tratamiento antirretroviral era considerado como algo positivo, y que a su vez lo seguían porque tenían motivaciones personales para estar bien, situaciones que tuvieron asociación estadística, y que han sido identificadas como aspectos que favorecen la adherencia al tratamiento y evita la aparición de reacciones adversas, ya que, cuando un individuo consume un medicamento con la predisposición de que les causará efectos negativos, tienen mayor tendencia a presentarlos y generar una conducta contraria a la esperada hacia el tratamiento ${ }^{8}$.

Tanto las cogniciones como los afectos, son aspectos que el profesional de enfermería debe incluir en forma rutinaria como parte de la valoración integral ofrecida a través de sus cuidados ${ }^{32}$, con el fin de generar las intervenciones que sean necesarias, las cuales pueden establecerse desde su autonomía o a través del vínculo con otros miembros del equipo de salud. Estos aspectos permiten contribuir con el bienestar de los usuarios y a su vez fomentan una práctica profesional alejada de la sobrecarga en el ejercicio del cuidar ${ }^{33}$.

En la presente investigación se reconoce que la cantidad de usuarios existentes en el programa representó una limitante para que los resultados pudiesen inferirse a otras poblaciones de adultos mayores en tratamiento para esta condición, de igual manera se evidenció la necesidad de incorporar el diseño cualitativo en futuros abordajes, sin embargo, la experiencia del análisis desde un modelo teórico de enfermería permitió establecer intervenciones con pertinencia disciplinar para este grupo de usuarios, aspecto que hace parte del valor social como criterio ético en el desarrollo de la investigación. 


\section{CONCLUSIONES}

Se puede establecer que los adultos mayores evaluados han logrado cumplir con la adherencia al tratamiento como conducta promotora de salud, aspecto que se ve favorecido por los factores personales de tipo psicológico, como son la ausencia de deterioro cognitivo y de alteraciones del ánimo, además de aspectos socioculturales como el contar con apoyo familiar $y / o$ de amistades.

La autoeficacia frente al consumo de medicamentos se fortalece debido a la baja percepción de barreras y el considerar como benéfico el consumo de los mismos, de igual manera, el contar con una o varias personas significativas en su entorno les motiva a mantener dicha conducta.

Pese la percepción de autoeficacia predominante, debe reconocerse como factores de riesgo de falla en el tratamiento tanto la mala adherencia reportada en algunos de los participantes, y el que la mayoría de ellos no lleve métodos para organizar sus medicamentos.

Es de destacar la importancia del profesional de enfermería en cuanto a la educación constante que debe brindar a los usuarios respecto de la terapia farmacológica en aspectos como: efectos adversos, beneficios, e importancia de mantener la adherencia al tratamiento farmacológico, lo cual permitirá mejorar sus percepciones y cogniciones; se debe además, fomentar la creación o activación de redes de apoyo, y la implementación de acciones que permitan mantener o mejorar la salud mental en cuanto a prevención de la depresión y estimulación cognitiva, como parámetros que facilitan el camino hacia la autoeficacia respecto al manejo de su condición de salud, lo cual se verá reflejado en su estabilidad clínica, siendo este un aporte importante desde la disciplina de enfermería a la salud pública.

Financiamiento: Dirección de Investigación de la Universidad De Los Lagos, Chile, DI-ULA No. 21/2017, R 13/17.

Conflicto de intereses: Los autores declaran no tener ningún conflicto de intereses.

\section{REFERENCIAS}

1. Ministerio de Salud de Chile. Guía Clínica AUGE: Síndrome de la inmunodeficiencia adquirida VIH/SIDA. Serie guías clínicas MINSAL. 2a ed. 2013; Ministerio de Salud. Chile.

2. Instituto de Salud Pública. Resultados confirmación de infección por VIH en Chile 2010-2015. Boletín Vigilancia de Laboratorio. Ministerio de Salud, 2016; 6(11): 1-13. Chile.

3. Ministerio de Salud de Chile. Informe Nacional: evolución de la infección por VIH/SIDA Chile 1984-2012. Rev. Chil. Infectol. 2015; 32(1): 17-43. http://dx.doi.org/10.4067/S0716-10182015000100003

4. Silva J, Werba Saldanha AAW. Vulnerabilidade e Convivência com o HIV/AIDS em Pessoas Acima de 50 Anos. Rev Mal-Estar e Subjetividade. 2012; 12(3/4): 817-53.

5. Ministerio de Salud de Chile. Orientaciones Técnica para la Atención de Salud de las Personas Adultas Mayores en Atención primaria. 1ed. Chile: Ministerio de Salud; 2014.

6. Hernández D, Abad Y, Valle EB. Linfocitos T CD4+ y carga viral en pacientes VIH/SIDA de la tercera edad que reciben tratamiento antirretroviral. Rev Cubana de Invest Bioméd. 2013; 32(2): 139-46.

7. Zamudio-Rodríguez A, Aguilar-Navarro S, Avila-Funes JA. Deterioro cognitivo en adultos mayores con VIH/ sida y síndrome de fragilidad. Gac Med Mex. 2017; 153: 598-607. http://dx.doi.org/10.24875/GMM.17002875

8. Organización Panamericana de la Salud (OPS). Experiencias exitosas en el manejo de la adherencia al tratamiento antirretroviral en Latinoamérica. Washington, D.C.: $O P S ; 2011,60$ p. 
9. Foresto JS, Melo ES, Costa CRB, Antonini M, Gir E, Reis RK. Adhesión a la terapia antirretroviral de personas con VIH/SIDA en un municipio del interior de São Paulo. Rev. Gaúcha Enferm. 2017; 38(1): 1. http://dx.doi.org/10.1590/1983-1447.2017.01.63158

10. Moya LP, Bermúdez-Román V, Bran Piedrahita L, Posada-Zapata IC. Influencia del estigma en torno al VIH en el acceso a los servicios de salud. Salud Pública Méx. 2015; 57(3): 252-9.

https://doi.org/10.21149/spm.v57i3.7564

11. Zuge SS, Primeira MR, Remor E, Magnago TSBS, Paula CC, Padoin SMM. Factores asociados a la adherencia al tratamiento antirretroviral en adultos infectados por VIH: estudio transversal. Rev Enferm UFSM. 2017; 7(4): 577-89. http://dx.doi.org/10.5902/2179769225657

12. Pino-Melgarejo M. Funcionamiento cognitivo en pacientes con VIH: algunas consideraciones teóricas. Rev Cubana Neurol Neurocir. 2015; 5 (Supl.1): 56-62.

13. Sakraida TJ. Modelo de Promoción de la Salud. En: Raile Alligood M, Marriner Tomey A. Modelos y Teorías en Enfermería. 8 ed. Barcelona: Elsevier; 2014. p 434-53.

14. Cadena JCE, Gonzáles YO. El cuidado de enfermería en pacientes con riesgo cardiovascular sustentado en la teoría de Nola J. Pender. Investig Enferm Imagen Desarr. 2017; 19(1): 107-21.

http://dx.doi.org/10.11144/Javeriana.ie19-1.ecep

15. Elm EV, Altman DG, Egger M, Pocock SJ, Gotzche PC, Vandenbroucke JP. The Strengthening the Reporting of Observational Studies in Epidemiology (STROBE) statement: guidelines for reporting observational studies. The Lancet. 2007; 370 (9596): 1453-7.

https://doi.org/10.1016/S0140-6736(07)61602-X

16. Carrasco-Aldunate $P$, Araya-Gutiérrez A, LoayzaGodoy C, Ferrer-Lagunas L, Trujillo-Guarda C, Pérez-Cortés $\mathbf{C}$, et al. Cómo entender la experiencia de personas que viven con VIH: implicaciones para la clínica y la investigación. Aquichan. 2013; 13(3): 387-95.

17. Vilató LF, Martín LA, Pérez IN. Adherencia terapéutica y apoyo social percibido en personas que viven con VIH/ SIDA. Rev Cubana Salud Pública. 2015; 41(4): 620-30.

18. Cordero MV. La adherencia al tratamiento antirretroviral en pacientes con VIH/SIDA y estrategias para su optimización. Revista Rol de Enfermería. 2017; 40(1): 2834.

19. Universidad Católica de Chile. Chile y sus Adultos Mayores: 10 años de la encuesta de calidad de Vida en la vejez. UC- Caja Los Andes. Chile: Centro UC Estudios de vejez y envejecimiento; 2017. 111 p.

20. Reis Rk, Melo ES, Castrighini CC, Galvão MTG, Toffano-Malaguti SE, Gir E. Prevalencia y factores asociados con síntomas depresivos en personas que viven con VIH/SIDA. Salud Ment. 2017; 40(2): 57-62. http://dx.doi.org/10.17711/sm.0185-3325.2017.008

21. Nhamba LA, Hernández EM, Bayarre HDV. Depresión en personas con VIH en dos municipios de Angola. Rev Cubana Salud Pública. 2014; 40(4): 276-88.
22. Cardona-Duque DV, Medina-Pérez OA, Herrera SMC, Orozco Gómez PAG. Depresión y factores asociados en personas viviendo con VIH/SIDA en Quindío, Colombia 2015. Rev Haban Cienc Méd. 2016; 15 (6): 941-95.

23. Mello SMP, Cardoso CP, Spiegelberg SZ, Ferreira TL, Santos ÉEP, Ribeiro MP. Terapia antirretroviral del VIH en adultos mayores de 50 años: prevalencia y clasificación de los no adherentes. Enf Glob. 2013; 12(31): 68-85.

24. Pinto MF, Gosuen GC, Vancini CR, Souza D,Assatag RE, Gonçalves ASB. Calidad de vida, perfil socioeconómico, conocimiento y actitud sobre sexualidad de "personas que viven" con el Virus de la Inmunodeficiencia Humana. Rev. Latino-Am. Enfermagem. 2015; 23(2): 192-9. http:// dx.doi.org/10.1590/0104-1169.3424.2542

25. Almeida JN, Oliveira AS, Ribeiro LS, Aparecida SA, Aparecida AM, Scatena TCV. Sida en adultos de 50 años y más: características, tendencia y difusión espacial del riesgo. Rev. Latino-Am. Enfermagem. 2014; 22(3): 35563. http://dx.doi.org/10.1590/0104-1169.3327.2424

26. Urra MS, Germán JLM. Caracterización de adultos mayores con polifarmacia evaluados en la consulta de geriatría. Rev Cubana Med Milit. 2014; 43(3): 285-92.

27. Cano-Gutiérrez C, Samper-Ternent R, Cabrera J, Rosselli D. Uso de medicamentos en adultos mayores de Bogotá, Colombia. Rev. Perú Med. Exp. Salud Pública. 2016; 33(3): 1-6.

https://doi.org/10.17843/rpmesp.2016.333.2292

28. Gómez NA, Caudevilla AM, Bellostas LM, Crespo MA, Velilla JM, Díez JM. Pluripatología, polifarmacia, complejidad terapéutica y uso adecuado de la medicación. Rev Clín Esp. 2017; (5) 289-95.

https://doi.org/10.1016/j.rce.2016.12.013

29. Alave JP, González J, Campos E, Rodríguez MM, Willig J, Echevarría J. Factores asociados a falla virológica en pacientes infectados con VIH que reciben terapia anti-retroviral en un hospital público del Perú. Rev. chil. infectol. 2013; 30(1): 42-8.

https://dx.doi.org/10.4067/S0716-10182013000100006

30. Pérez ER, Soler YMS, Hung YF, Rondón MZ. Programa educativo para favorecer la adherencia terapéutica en pacientes con tratamiento antirretroviral. AMC. 2016; 20 (2): 177-87.

31. García-Reza C, Landeros ML, Gollner RZ, Solano-Solano G, Alvarado LA, Morales MP. Rol socioeconómico y la adhesión al tratamiento de pacientes con hipertensión arterial - contribución de enfermería. Rev Cuid. 2012; 3(1): 280-6. http://dx.doi.org/10.15649/cuidarte.v3i1.22

32. Corrêa PPS, Andrade M, Santos ÉI, Espírito FHS, Souza ALB, Teixeira PA. Evidencia científica de enfermería acerca del HIV/AIDS entre ancianos: una revisión de la literatura. Rev baiana enferm. 2015; 29(3): 278-89.

33. Lopera MA, Forero C, Paiva LE, Cuartas VM. El quehacer cotidiano de la enfermera significa soportar la carga. Rev Cuid. 2016; 7(2): 1262-70.

http://dx.doi.org/10.15649/cuidarte.v7i2.320 